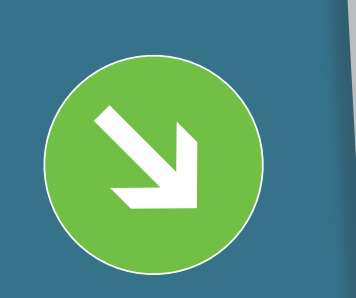

Ключевые слова:

САМ-система,

ЧПУ, управляю-

щая программа,

постпроцессор,

токарно-фрезерная обработка

\title{
ОСОБЕННОСТИ
}

\section{И ПРЕИМУЩЕСТВА СИСТЕМЫ АDЕМ В ОБЛАСТИ СОЗДАНИЯ УПРАВЛЯЮЩИХ ПРОГРАММ ДЛЯ СТАНКОВ С ЧПУ}

\author{
ЧАСТЬ $2^{*}$
}

\section{Даниил ЗИНЧЕНКО}

В данной статье представлен опыт реализации CAM-системы ADEM на различных предприятиях в части программирования чПУ-обработки.

\section{ОБРАБОТКА ПОДНУТРЕНИЙ НА ТЕЛАХ ВРАЩЕНИЯ}

B системе ADEM существуют специальные возможности по обработке тел вращения с канавками и выточками на периферической поверхности. При задании токарной обработки существует возможность отключения опции обработки всякого рода поднутрений, которые предполагают уклонение движения резца от прямолинейного движения на проход. Это позволяет лишний раз не тратить рабочее время технолога и не обозначать контуры заготовки, если нам необходимо начерно снять как можно больше материала и подготовить базы под чистовую обработку. В дальнейшем, задав опцию обработки поднутрений и выбрав резец с углом при вершине, к примеру $35^{\circ}$, можно начисто обработать все имеющиеся на поверхности тела вращения канавки (рис. 13).

\section{ФРЕЗЕРОВАНИЕ ПАЗОВ И ОКОН НА ЦИЛИНДРИЧЕСКИХ ПОВЕРХНОСТЯХ}

В случае если на детали имеются пазы или окна на периферической поверхности с каким-либо фигурным профилем, в ADEM существуют очень простые инструменты для задания обработки таких пазов или окон. Геометрически любой контур на поверх-

*Начало статьи опубликовано в журнале «СТАНКОИНСТРУМЕНТ», 2019, № 1 . ности цилиндра можно развернуть на плоскость. То есть фактическую токарно-фрезерную обработку паза или окна в 4-х координатах (XYZC) можно осуществить как 2,5D-координатное фрезерование кон-
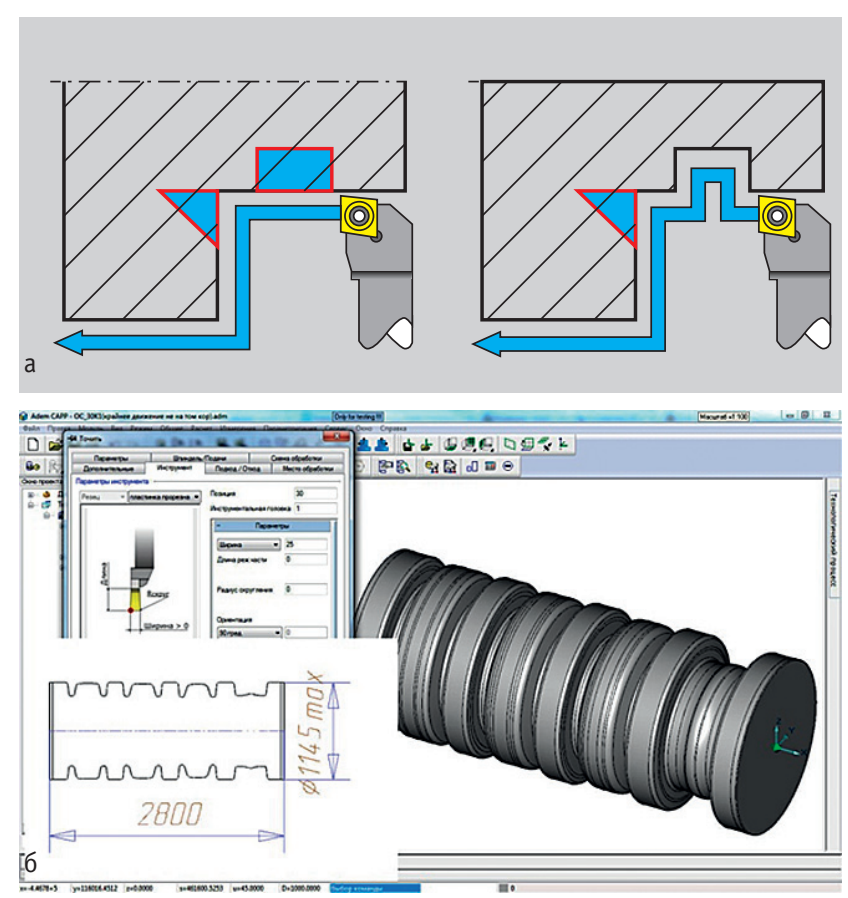

Puc. 13. Обработка канавок на токарных станках в ПО ADEM 

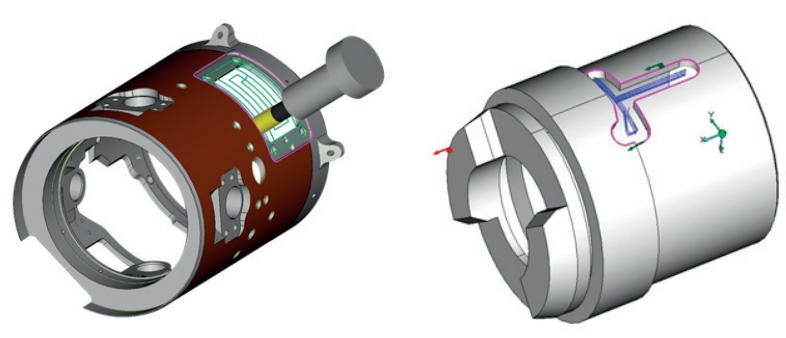

Puc. 14. Обработка пазов и окон на цилиндрических поверхностях

тура на плоскости. С помощью своих внутренних алгоритмов система ADEM делает такую развертку контура окна на плоскость и, задав образующую линию, которая определяет фактическую цилиндрическую поверхность детали, можно выполнить обработку нашего паза или окна с помощью опции системы «Фрезерование 2,5D». Это позволяет предприятиям осуществлять обработку в 4-х координатах, имея лицензию ADEM 2,5D (рис. 14).

\section{5-КООРДИНАТНАЯ ОБРАБОТКА В АDЕМ}

Далее коснемся возможностей ADEM при 5-координатной обработке. При обработке поверхностей часто возникает необходимость корректировки положения инструмента относительно обрабатываемой поверхности. На рис. 15 наглядно видно, в каких случаях это может понадобиться - при фрезеровке выпуклых поверхностей фрезами с пластинками,

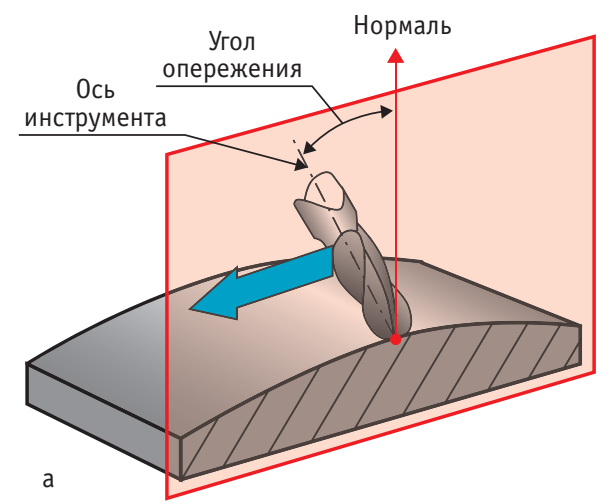

Puc. 15.

Углы отклонения и опережения при обработке поверхностей

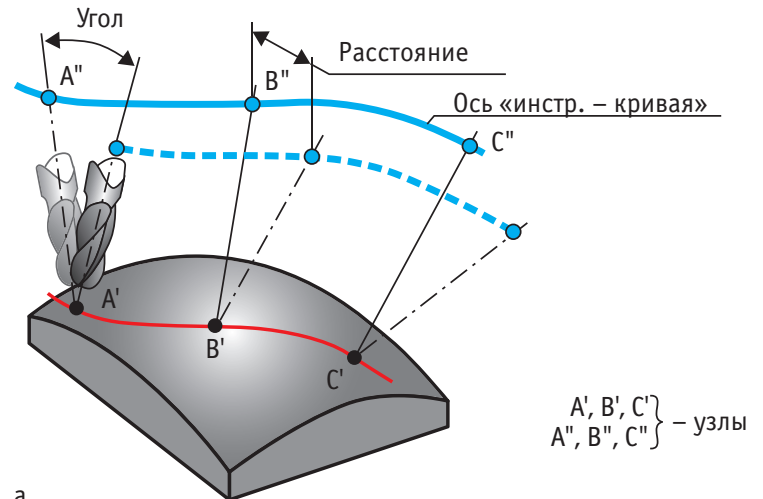

A'A", B'B", C'C" - прямые соединения узлов

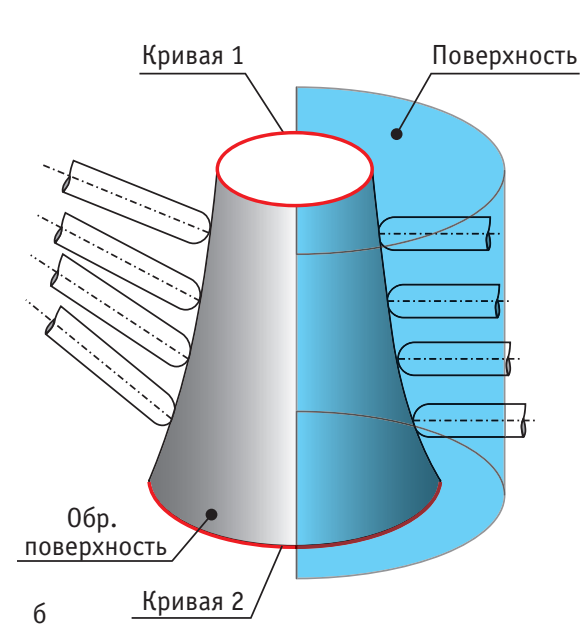

Puc. 16.

Задание обработки с помощью кривых и поверхностей

когда в центре фрезы образуется участок без режущих элементов. В таких случаях смещение инструмента в продольном или поперечном направлении, позволяет уйти от образования подобных зон с нулевой скоростью резания. В этих случаях используется управление осью инструмента по угловым осям. Это использование так называемых углов отклонения и углов опережения, в результате которого инструмент искусственно как бы заваливается вперед по ходу движения.

Для более сложных случаев может быть задействовано управление осью инструмента с использованием дополнительных кривых или поверхностей (рис. 16). В этом случае кончик инструмента идет по обрабатываемой поверхности, а его хвостовая часть движется вдоль указанной кривой. Яркий пример - обработка моноколес.

При обработке деталей типа «моноколесо» значительное время затрачивается на черновую обработку. Это происходит вследствие того, что моноколеса чаще всего изготавливают из титановых сплавов, что влияет на подход к обработке. При традиционной технологии черновой обработки таких деталей материал выбирается продольными проходами с небольшими снимаемым припуском и подачами. Увеличить подачу при такой схеме обработки существенно не получится в свя- 

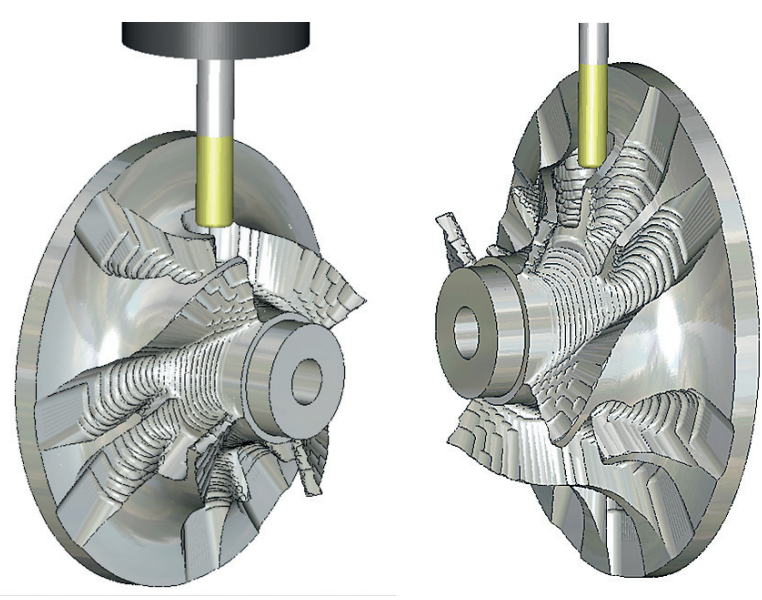

Puc. 17. Плунжерное черновое фрезерование при обработке моноколес

зи с высоким коэффициентом износа инструмента. Уменьшить затраты на этом этапе можно посредством применения плунжерного фрезерования (рис. 17).

В системе ADEM допустимо использование плунжерного фрезерования не только в 3-осевом режиме, но и совместно с осями вращения.

Этот вид обработки в 2-3 раза повышает подачу за счет использования осевого врезания инструмента и существенно увеличивает размер снимаемого припуска. При выполнении проходов система отслеживает положение инструмента относительно обрабатываемых поверхностей, что позволяет уже на первом этапе грубой черновой выборки подготовить криволинейные поверхности к дальнейшей обработке.

При чистовой обработке лопастей моноколеса основная проблема, с которой сталкивается технолог, это управление осью инструмента непосредственно на поверхности лопасти и на радиусе скругления.

Как известно, поверхность лопасти представляет собой линейчатую поверхность и теоретически может быть обработана боковой частью обычной концевой фрезы за один проход. В реальности же кривизна втулки и искривление лопасти делают такой подход к обработке невозможным. В производстве, как правило, поверхность лопасти обрабатывают сферическим или сфероконическим инструментом продольными проходами с постоянным или переменным углом отклонения от поверхности. Но в этом случае при переходе через радиус скругления носика лопасти (рис. 18) ось инструмента делает резкий рывок для того, чтобы на следующей поверхности обеспечить заданный угол отклонения. Для устранения такого рода ситуаций в системе ADEM дополнительно к параметрическим средствам управления осью инструмента существует возможность управлять наклоном инструмента с помощью кривых и поверхностей.

Традиционный подход к обработке турбинных лопаток заключается в следующем: непрерывная

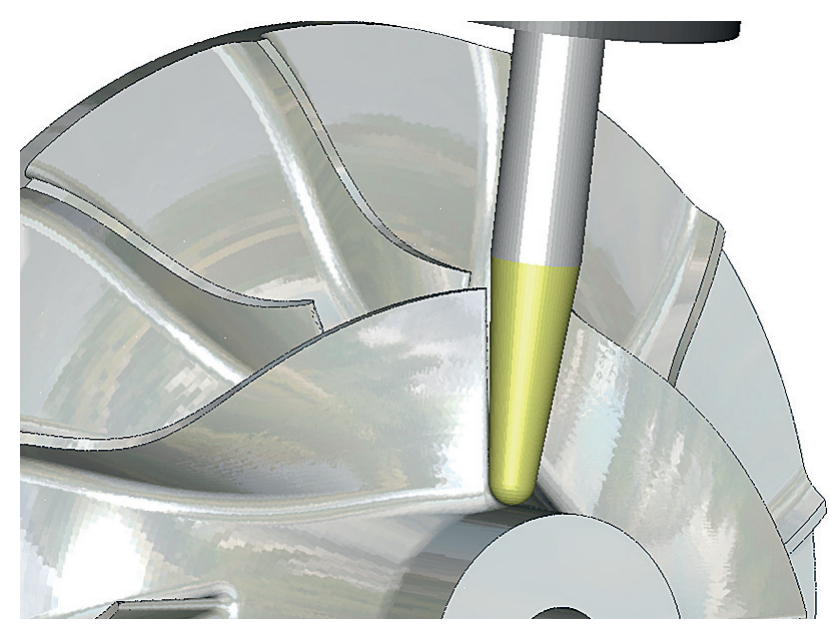

Puс. 18. Обработка носика лопасти

обработка по спирали пера лопатки и после этого доработка замковой части. Но этот подход имеет ряд недостатков:

$\rightarrow$ требует использования сферического инструмента, так как стандартный концевой инструмент «нахлопывается» торцем на припуск при переходе с поверхности корыта на поверхность горба, что приводит к поломке;

$\rightarrow$ грубая огранка на радиусах скругления даже при жесткой аппроксимации. Причем чем больше кривизна пера, тем более существенными будут дефекты. Кроме того, из-за очень коротких перемещений в кадрах значительно уменьшается реальная подача, что приводит к ускорению износа («засаливанию») инструмента.

В результате этого ухудшается качество обработки и возрастает доля слесарной доводки.

Для получения качественных поверхностей в ADEM присутствует возможность разделять зоны обработки и использовать для каждой зоны оптимальный инструмент.

Обработку поверхностей корыта и радиусов скругления необходимо производить сферическим инструментом с максимально возможным боковым упреждением продольными проходами (рис. 19). Это позволяет:

$\rightarrow$ исключить обработку с нулевой скоростью резания; $\rightarrow$ увеличить длину перемещений в кадре;

$\rightarrow$ обработать радиус скругления с точностью станка.

Обработку горба лопатки необходимо производить концевой фрезой с радиусом на торце поперечными проходами с минимальным, но отличным от нуля продольным угловым упреждением. Это позволяет получить практически зеркальную поверхность.

Однако существуют проблемы не только при создании оптимальных траекторий обработки и задании режимов резания, но и при разработке пост- 


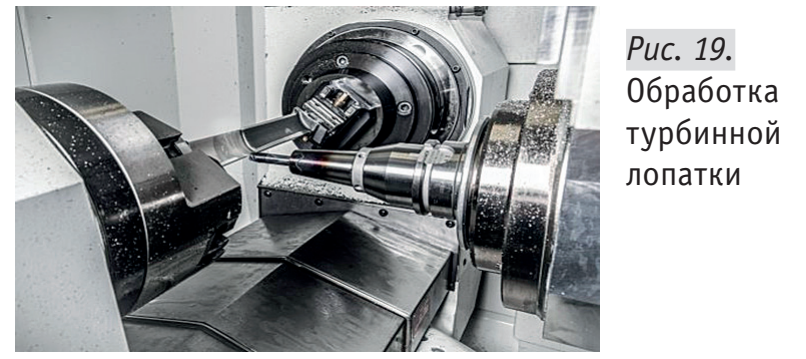

процессора под определенный станок с ЧПУ. Например, предприятия нередко приобретают многокоординатные станки с урезанным функционалом по управлению центром инструмента в системе ЧПУ (RTCP). Это порой объясняется тем, что продавцы станков с ЧПУ просто не афишируют определенную техническую информацию - открытие в функционале системы ЧПУ команды управления центром инструмента G43.3 может привести к существенному повышению стоимости станка. Специалисты Группы компаний ADEM способны разработать постпроцессоры, заменяющие недостающие функции набором открытых команд для управления положением инструмента.

Одна из новых задач, с которой сегодня могут столкнуться поставщики САМ-систем, состоит в реализации работы с контрольно-измерительными циклами и необходимостью вывода резуль-

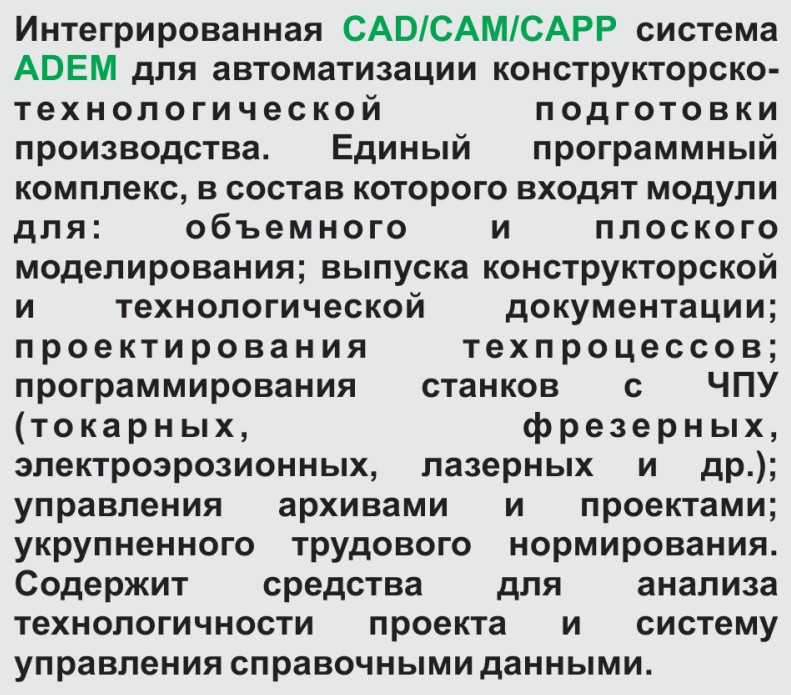

Интегрированная CAD/CAM/CAPP система ADEM для автоматизации конструкторскотехнологической подготовки производства. Единый программный комплекс, в состав которого входят модули для: объемного и плоского моделирования; выпуска конструкторской и технологической документации; проектирования техпроцессов; программирования станков с ЧПу (токарных, фрезерных, электроэрозионных, лазерных и др.); управления архивами и проектами; укрупненного трудового нормирования. Содержит средства для анализа технологичности проекта и систему управления справочными данными.

\section{Группа компаний ADEM}

107497, Москва:

ул. Иркутская, д. 11 тел: +(7) 495 462-0156,

$+(7) 495502-1341$

e-mail: moscow@adem.ru
426003, Ижевск:

ул. Красноармейская, д. 69 тел: +(7) 3412 522-341, + (7) $3412522-433$ e-mail: izhevsk@adem.ru татов измерения в файл отчета. Эта задача тоже лежит в плоскости постпроцессирования. Один из наших клиентов приобрел 5-координатный вертикально-фрезерный станок с измерительными датчиками фирмы Renishaw (рис. 20). Номенклатура производства состояла из мелкогабаритных деталей, которых на рабочем столе станка могло разместиться несколько десятков. И, соответственно, возникла задача осуществлять межоперационный обмер каждой детали непосредственно на столе станка. Эта задача была успешно решена - создан отдельный постпроцессор с программированием измерительных циклов датчика и генерированием файла отчета.

Работа с циклами соответствующей системы ЧПУ позволяет, как известно, значительно сократить УП, упростить процесс задания и контроля параметров обработки. Также короткая программа легче считывается системой ЧПУ, которая перед наступлением следующего кадра УП успевает дать соответствующую команду на приводы станка. Интересная задача - заставить САМ-систему формировать УП с учетом специальных функций интерфейсов ShopTurn и ShopMill компании Siemens. Сложность в данном случае заключается в необходимости вывода в УП множества служебных команд. Хотя и эта задача вполне успешно решается путем разработки специального постпроцессора.
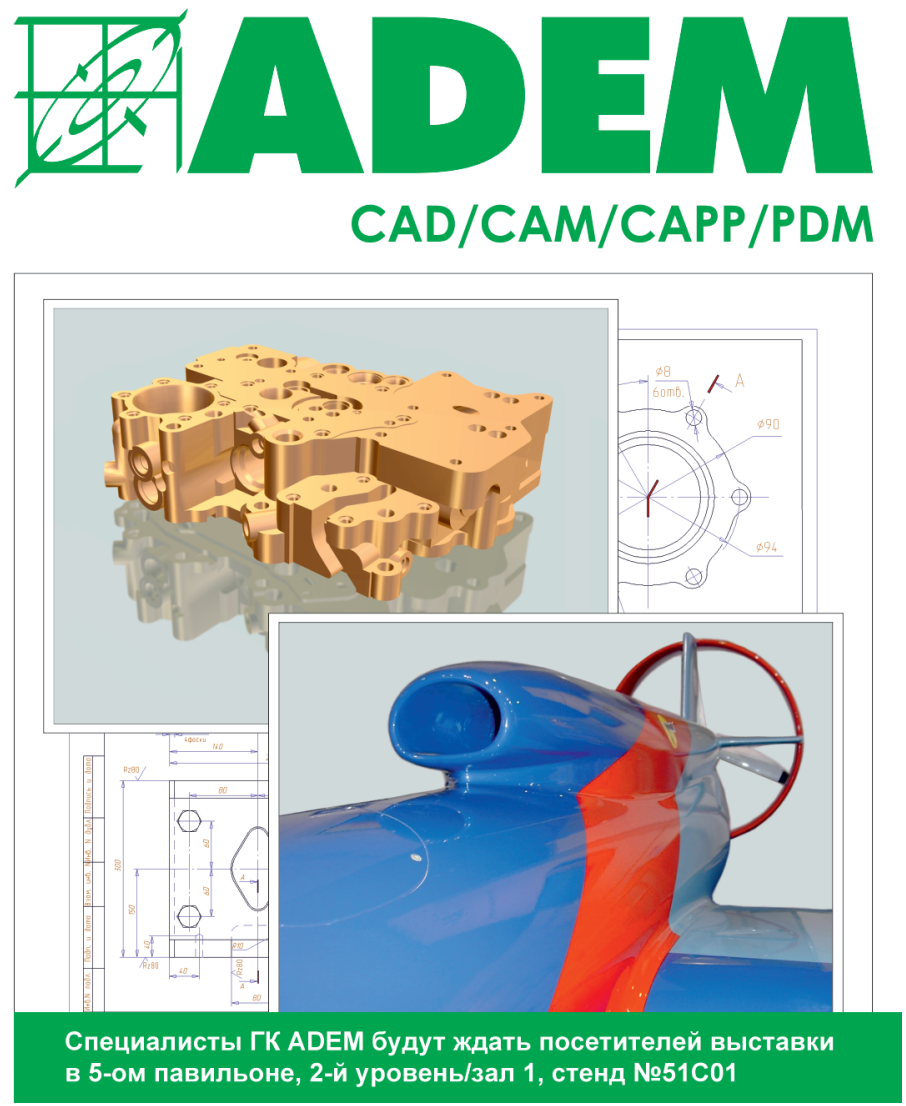


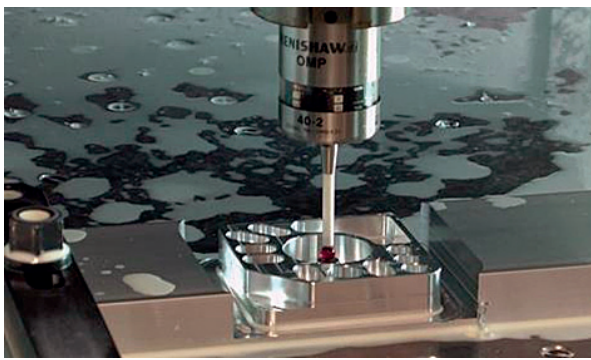

Puc. 20.

Процесс измерения

детали системой Renishaw

\section{ADEM САМ EXpert - МОДУЛЬ АВТОМАТИЧЕСКОГО} ФОРМИРОВАНИЯ МАРШРУТА ЧПУ-ОБРАБОТКИ

Система ADEM имеет в своем составе уникальный компонент, позволяющий в автоматическом режиме получать маршрут ЧПУ-обработки детали, - модуль ADEM CAM Expert.

Функционал модуля ADEM CAM Expert позволяет распознавать обрабатываемые элементы 3D-модели детали и предлагает технологу-программисту маршрут обработки этой детали на станке. Модуль ADEM CAM Expert для каждого из этих элементов в автоматическом режиме рассчитывает наиболее оптимальную траекторию обработки, осуществляет подбор режущего инструмента в зависимости от геометрии обрабатываемых поверхностей, а также рассчитывает режимы резания для выбранного инструмента.

При использовании модуля ADEM CAM Expert технологу-программисту предоставляется возможность получения маршрута обработки детали на станках с ЧПУ в автоматическом режиме, что позволяет сократить общее время технологической подготовки обработки детали.

Основные этапы работы модуля ADEM CAM Expert:

$\rightarrow$ загрузка 3D-модели обрабатываемой детали (рис. 21);

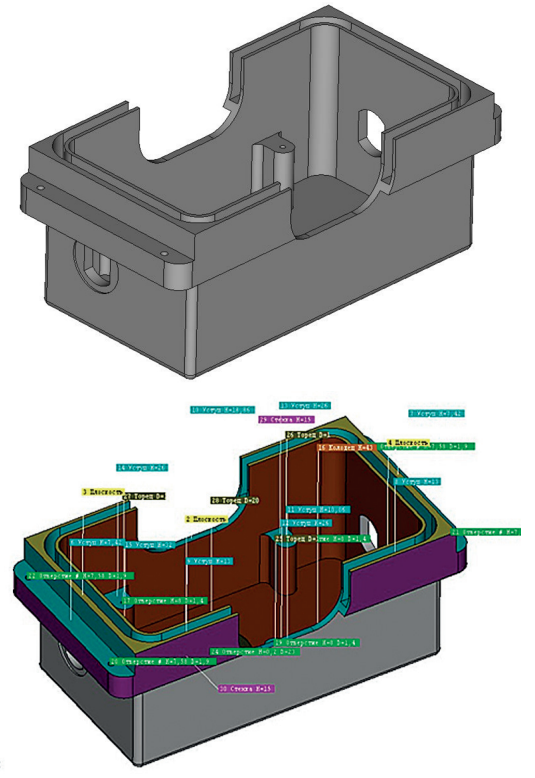

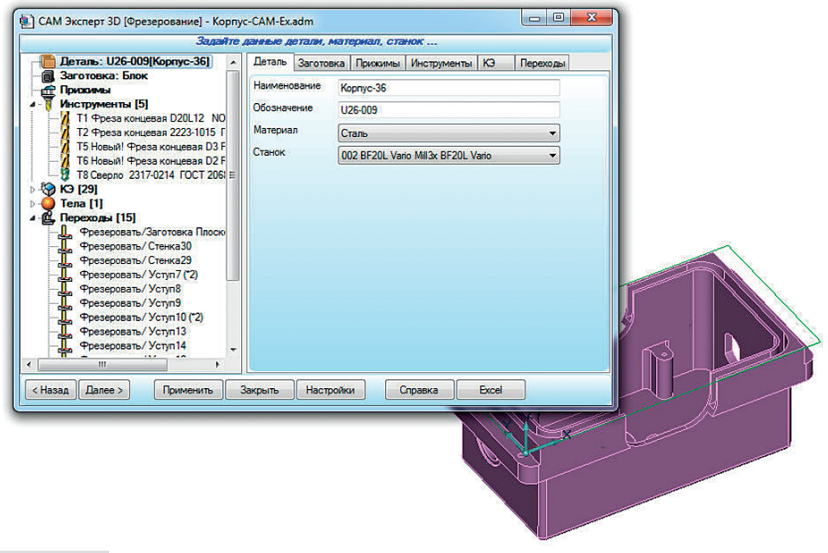

Puc. 23. Подборка маршрута обработки согласно по конструктивным элементам

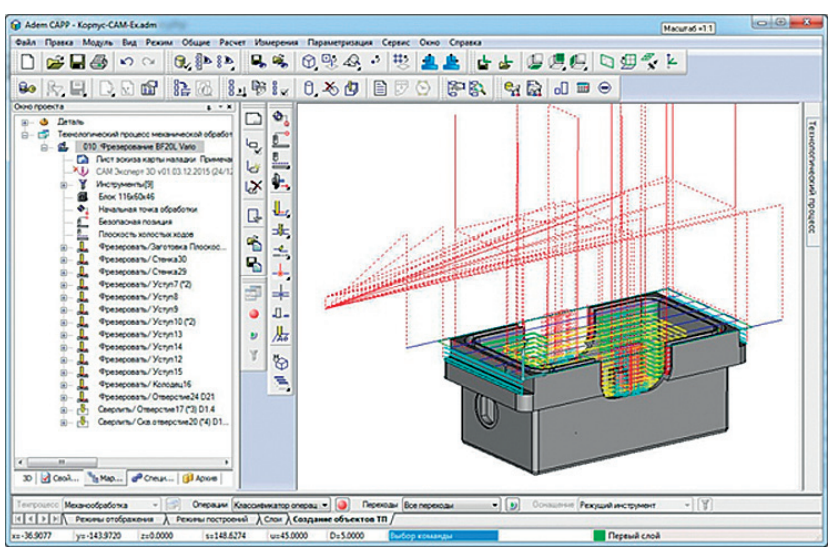

PUC. 24. Построение траекторий обработки согласно маршрута

$\rightarrow$ анализ геометрии детали и представление электронной модели детали с визуализацией конкретных конструктивных элементов (рис. 22);

$\rightarrow$ диалоговое окно ADEM CAM Expert для просмотра и коррекции параметров спроектированного в автоматическом режиме маршрута обработки детали (рис. 23);

$\rightarrow$ расчет траектории движения инструмента с учетом выбранного типа оборудования с ЧПУ (рис. 24);

$\rightarrow$ моделирование обработки внутренними средствами модуля ADEM CAM для оценки качества сформированной траектории обработки и внесения корректировок в составленный маршрут обработки (рис. 25).

При необходимости корректировка составленного маршрута может производиться и во внешних приложениях, например в формате таблицы MS Excel с использованием макросов. Все внесенные коррективы при этом отображаются и в дереве технологического процесса в модуле ADEM CAM Expert (рис. 26). 

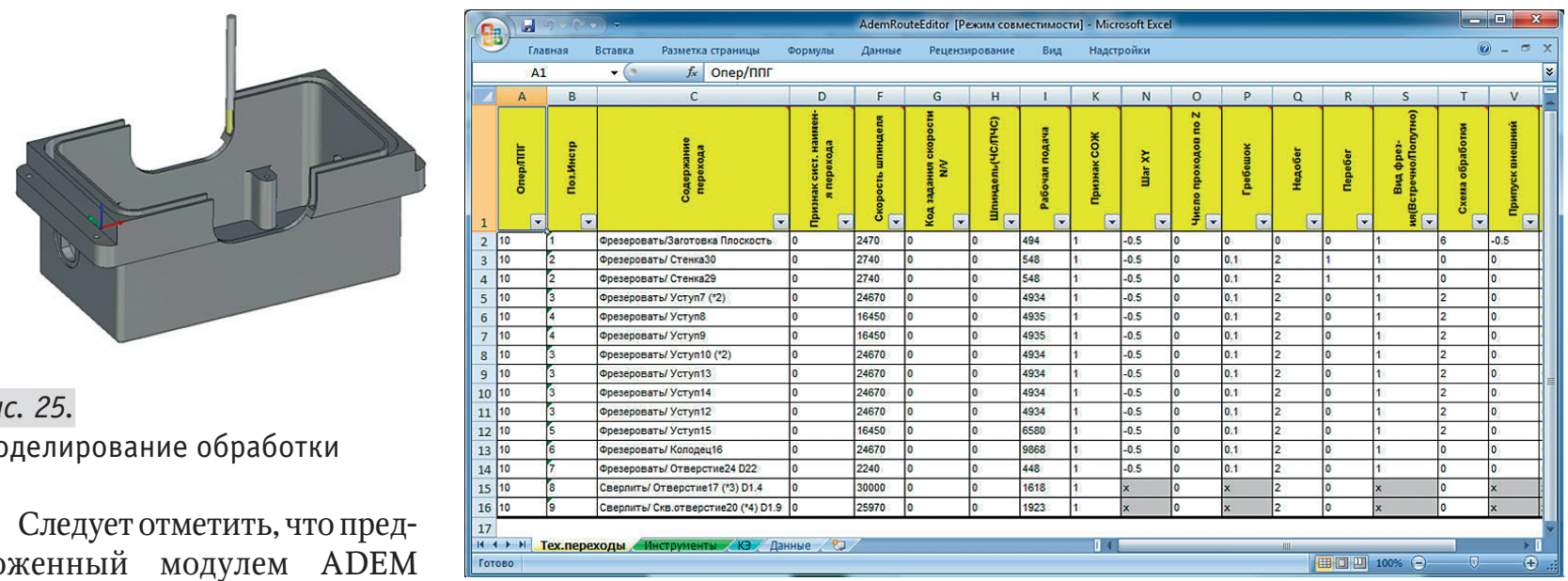

Puc. 25.

Моделирование обработки

Следует отметить, что предложенный модулем ADEM CAM Expert маршрут обработки детали, безусловно, может быть отредактирован програм-

Puc. 26. Маршрут обработки в виде таблиц MS Excel мистом. Изменения могут быть внесены в стратегию обработки конструктивного элемента, в режимы резания, может быть изменен или добавлен режущий инструмент взамен автоматически подобранного из имеющихся баз данных по режущему инструменту.

Как показывает практика, внедрение модуля ADEM CAM Expert ускоряет процесс технологического проектирования обработки детали на оборудовании с ЧПУ в среднем на $20 . . .30 \%$.
Таким образом, в данной статье мы рассмотрели как основные технические особенности проектирования ЧПУ-обработки в ПО ADEM, так и практическую реализацию этих особенностей, которая подтверждена многолетним опытом успешных внедрений.

\section{ЗИНЧЕНКО ДанИил -}

ведущий специалист Группы компаний ADEM

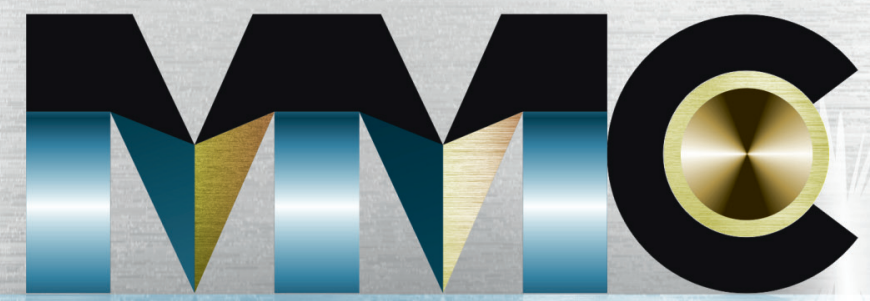

4-6АЕКАНЬ 2019

19-я межАународная специализированная выставка оборудования, приборОв И ИНСтрУментов Аля машиностроительной,

метамлообрабатывающей и сварочной отраслей промышленности

\section{МАШИНОСТРОЕНИЕ МЕТАММООБРАБОТКА CBAPKA}

\section{КАЮЧЕВОЕ СОБЫТИЕ ОТРАСАИ}

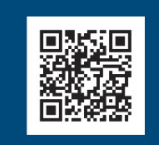

Забронировать стенA

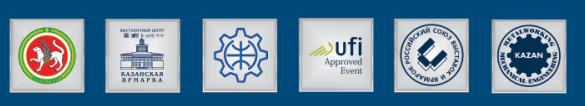

$16+$
ОРГКОМИТЕТ ВЫСТАВОК Россия, 420059, г. Казань, Оренбургский тракт, А. 8, Тел/факс: (843) 20-29-03, 202-29-92

ОАО "Казанская ярмарка" www.expomach.ru, www.expokazan.ru E-mail: expo-kazan@mail.ru 